\title{
Hadron properties from QCD bound-state equations: A status report
}

\author{
Reinhard Alkofer ${ }^{1} \quad$ Gernot Eichmann ${ }^{1,2}$, Andreas Krassnigg ${ }^{1}, \quad$ Diana Nicmorus ${ }^{1}$ \\ 1 Institut für Physik, Karl-Franzens-Universität Graz, 8010 Graz, Austria \\ 2 Institute for Nuclear Physics, Darmstadt University of Technology, 64289 Darmstadt, Germany
}

\begin{abstract}
Employing an approach based on the Green functions of Landau-gauge QCD, some selected results from a calculation of meson and baryon properties are presented. A rainbow-ladder truncation to the quark Dyson-Schwinger equation is used to arrive at a unified description of mesons and baryons by solving BetheSalpeter and covariant Faddeev equations, respectively.
\end{abstract}

Key words functional approaches; Bethe-Salpeter equation; covariant Faddeev equation

PACS 11.10.St, 12.38.Lg, 14.20.Dh

\section{Motivation:}

\section{Why Functional Approaches to QCD?}

The central aim of the studies reported here is to develop a QCD-based description of the structure of hadrons in terms of quarks and gluons. Theoretical issues such as confinement, dynamical breaking of chiral symmetry and the formation of relativistic bound states can be understood and related to the properties of QCD's Green functions. Significant progress within functional approaches has recently been achieved especially in Landau gauge, see e.g. refs. $[1, \underline{2}, \underline{3}, \underline{\underline{n}}$. Hereby it should be noted that different pictures of confinement are not necessarily mutually exclusive but may describe different facets of this phenomonen, see ${ }^{[5]}$ and references therein. The advantage of employing Green function methods is given by the fact that these Green functions provide the input for QCD's bound state equations which, in turn, can be used to calculate hadron properties. The Green functions of elementary fields are determined by Dyson-Schwinger equations, Exact Renormalization Group equations, and/or lattice calculations. It should be noted that dynamical breaking of chiral symmetry leads to the generation of quark masses and scalar quark-gluon interactions $[3,6]$.

Any numerical solution of QCD's bound state equations, i.e. the Bethe-Salpeter equation for mesons and the Faddeev equation for baryons, requires Green functions of quarks and gluons as input, which in turn necessitates a truncation of the corresponding functional equations. The results which will be presented in this status report build upon a rainbow-ladder truncation, i.e. a dressed-gluon exchange, for mesons and baryons in a unified approach $[\underline{7}, \underline{8}, \underline{9}, \underline{10}, \underline{11]}$. A symmetry-preserving extension to more realistic kernels is on its way for mesons, see ${ }^{[12]}$ and references therein. For baryons as three-quark states, the covariant Faddeev equation in rainbow-ladder truncation has been solved only recently ${ }^{[13]}$.

\section{Bound state equations and rainbow-ladder truncation}

Hadrons, being bound states of quarks, appear as poles in the $q \bar{q}$ and $q q q$ scattering matrices. Hadron properties can be extracted upon solving bound-state equations which are valid at these poles and need the elementary QCD Green functions as input:

$$
\Psi=\widetilde{K}_{(\mathrm{n})} \Psi .
$$

Here $\Psi$ is the bound-state amplitude defined on the hadron's mass shell, and $\widetilde{K}_{(2)}=K_{(2)} S S, \widetilde{K}_{(3)}=$ $K_{(3)} S S S$ are the renormalization-group invariant products of the respective kernel $K_{(\mathrm{n})}$ with two or three dressed quark propagators.

These fully Poincaré-covariant bound-state equations provide a tool to calculate experimentally accessible hadron observables, e.g. meson and baryon mass spectra, decay constants, scattering processes, and 


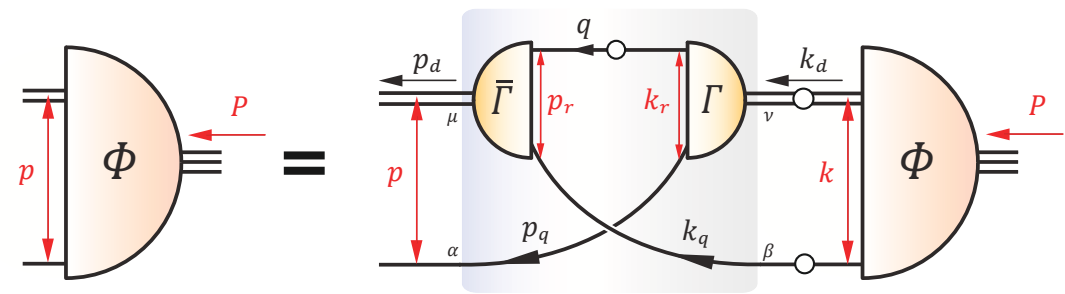

Fig. 1. The quark-diquark BSE in pictorial form.

electromagnetic properties such as form factors, magnetic moments and charge radii (see e.g. Refs. $\left.{ }^{[8,14}\right]$ and references therein). To proceed with the numerical solution of a covariant bound-state equation like the Bethe-Salpeter or the Faddeev equation, one needs to specify all ingredients: the interaction kernels and the dressed quark propagator which appear in these integral equations. These Green functions can be determined either from functional equations and/or lattice calculations. In addition, they are related via Slavnov-Taylor and Ward-Takahashi identities. With respect to the pion, the would-be Goldston boson of chiral symmetry, the axial-vector Ward-Takahashi identity plays a special role. If it is satisfied by the interaction kernels in related equations, this correctly implements chiral symmetry and its dynamical breaking, leading e.g. to a generalized Gell-Mann-Oakes-Renner relation which is valid for all pseudoscalar mesons and all current-quark masses $[15,16]$. In particular, the pion is massless in the chiral limit independent of the details of the interaction.

Although the structure of the kernel is restricted by the above mentioned identities, to construct a symmetry-preserving truncation of the bound-state equations is a non-trivial task. The rainbow-ladder truncation provides such a symmetry-preserving scheme. Instead of interaction kernels being functions of the quark and gluon momenta one needs a functional form for the interaction as a function of the gluon momentum only. The non-perturbative dressing of the gluon propagator and the quark-gluon vertex are "absorbed" into an effective coupling for which we adopt a widely-employed ansatz $\left.{ }^{[17}, 18\right]$. It reproduces the logarithmic decrease of QCD's oneloop perturbative running coupling at high momenta, and its infrared contribution is parametrized by an infrared scale and a dimensionless parameter (for details, see $\left.{ }^{[18]}\right)$. Especially, it yields the non-perturbative enhancement at intermediate gluon momenta necessary to generate dynamical chiral symmetry breaking, and hence a constituent-quark mass scale.
We emphasize here that the input of the Faddeev equation is completely specified at the beginning with all parameters already fixed from a few meson properties. In addition, we want to note that the resulting quark propagator is in very good agreement with corresponding results of fully coupled Dyson-Schwinger, resp., Functional Renormalization Group equations and of lattice calculations.

\section{Baryons: Quark-diquark model}

As the amplitudes resulting from a fully relativistic Faddeev equation are of high complexity it is natural to look first for an approximation keeping Poincaré invariance and the relation to QCD Green functions. A well-studied approximation of this type is based on using diquark correlations as effective degrees of freedom.

The motivation for studying diquark correlations has been the observation of a strong attraction in the $S U(3)_{C}$ antitriplet $q q$ channel, e.g. in lattice $[19,20,21,22,23,24]$ and Bethe-Salpeter $[25,26]$ studies. Such an attraction has also been proposed to explain missing exotic states in the hadron spectrum and the masses of light scalar mesons $\left.{ }^{[27}, 28\right]$. Further support for the diquark concept has been provided by a study of diquark confinement in Coulomb-gauge QCD ${ }^{[29]}$.

These arguments lead to the assumption that correlations between two quarks provide the dominant attraction not only in meson but also in baryon channels. Consequently, the quark-diquark model traces the nucleon's binding to the intrinsic formation of colored scalar- and axialvector diquarks. It treats such two-quark correlations as a separable pole sum in the $q q$ scattering matrix which leads to a description of baryons as bound states of effective quarks and diquarks. In this way the covariant Faddeev equation (see Section 4) is simplified to a two-body boundstate equation (see Fig. 1) while full Poincaré covariance is maintained.

Nucleon and $\Delta$ properties have been studied in a quark-diquark model with parametrized ingredients, 


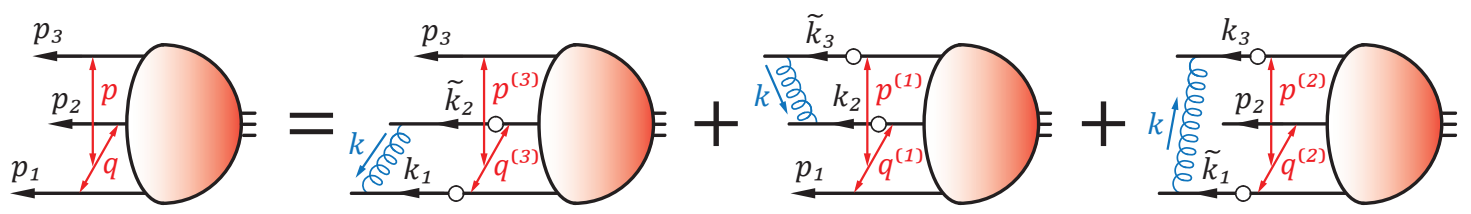

Fig. 2. Faddeev equation (2) in rainbow-ladder truncation.

see e.g. $[30,31,32,33]$ and references therein. The approach was subsequently extended to determine the dynamics of $0^{+}$and $1^{+}$diquarks from their underlying quark and gluon constituents $[\underline{\underline{8}}, \underline{9}, \underline{10}, \underline{11}, \underline{34}$ where parametrizations for the diquark amplitudes were removed and replaced by solutions of the corresponding diquark Bethe-Salpeter equations.

The identification of colored diquarks as poles in the $q q$ scattering matrix is possible within a rainbow-ladder truncation: one obtains timelike $0^{+}$, $1^{+}, \ldots$ diquark poles. The corresponding mass scales play an important role in the description of light baryons. These poles, however, correspond to unphysical asymptotic states and disappear from the spectrum when going beyond rainbow-ladder. The obtained rainbow-ladder diquark masses exhibit large sensitivities to an effective width parameter of the rainbow-ladder interaction kernel, a feature which has previously been observed in Ref. ${ }^{[25]}$.

The results for nucleon and $\Delta$ masses employing the quark-diquark bound state equation (Fig. 1) are shown in the left panel of Fig. 3 together with a selection of lattice results. The corresponding abscissa values $m_{\pi}^{2}$, as well as $m_{\rho}$, are obtained from the pseudoscalar and vector-meson Bethe-Salpeter equations in one consistent calculation. The findings are qualitatively similar to those for $m_{\rho}$ : setup A, where the coupling strength is adjusted to the experimental value of $f_{\pi}$, agrees with the lattice data. This behaviour can be understood in light of a recent study of corrections beyond rainbow-ladder truncation which suggests a near cancellation in the $\rho$-meson of pionic effects and non-resonant corrections from the quarkgluon vertex ${ }^{[12]}$. Setup B provides a description of a quark core which on purpose overestimates the experimental values (see the discussion below) while it approaches the lattice results at larger quark masses.

\section{Baryons: covariant Faddeev equation}

A formalism to treat the three-body bound-state problem through the analogue of the two-body Bethe-
Salpeter equation was formulated in Refs. ${ }^{[35,36]}$, see ${ }^{[37]}$ for an overview. The corresponding covariant Faddeev equation describes the baryon as a bound state of three spin- $1 / 2$ valence quarks (cf. Fig. 2):

$$
\Psi=\widetilde{K}_{(3)} \Psi, \quad \widetilde{K}_{(3)}=\widetilde{K}_{(3)}^{\mathrm{irr}}+\sum_{a=1}^{3} \widetilde{K}_{(2)}^{(a)},
$$

where the interaction kernel $\widetilde{K}_{(3)}$ comprises a threequark irreducible contribution and the sum of permuted two-quark kernels whose quark-antiquark analogues appear in a meson Bethe-Salpeter equation. The subscript $a$ denotes the respective accompanying spectator quark.

The QCD Green functions once more provide a way to embed this equation in a consistent quantumfield theoretical setup. Its dynamical ingredients can then be treated in perfect correspondence with studies of quark and meson properties. A solution of the equation relies upon knowledge of the dressed quark propagator and the three-quark kernel. A numerically solvable form of this equation is based on the specification and decomposition of the Poincarécovariant baryon amplitude.

Recently a fully Poincaré-covariant computation of the nucleon's Faddeev amplitude was performed in such a unified treatment $[13,38]$. The assumption of dominant quark-quark correlations motivates the omission of the three-body irreducible contribution from the full three-quark kernel in Eq. (2). The numerical solution of the Faddeev equation is then performed after truncating the interaction kernel to a dressed gluon-ladder exchange between any two quarks, thereby making a direct comparison with corresponding meson studies meaningful. Further insight into the structure of baryons is provided by contrasting the results to those of investigations of baryons in the quark-diquark model.

The resulting nucleon mass $M_{N}$ and its evolution with $m_{\pi}^{2}$ is plotted in the right panel of Fig. 3. A comparison to the consistently obtained quark-diquark model result exhibits a discrepancy of only $\sim 5 \%$. This surprising and reassuring result indicates that a description of the nucleon as a superposition of scalar 

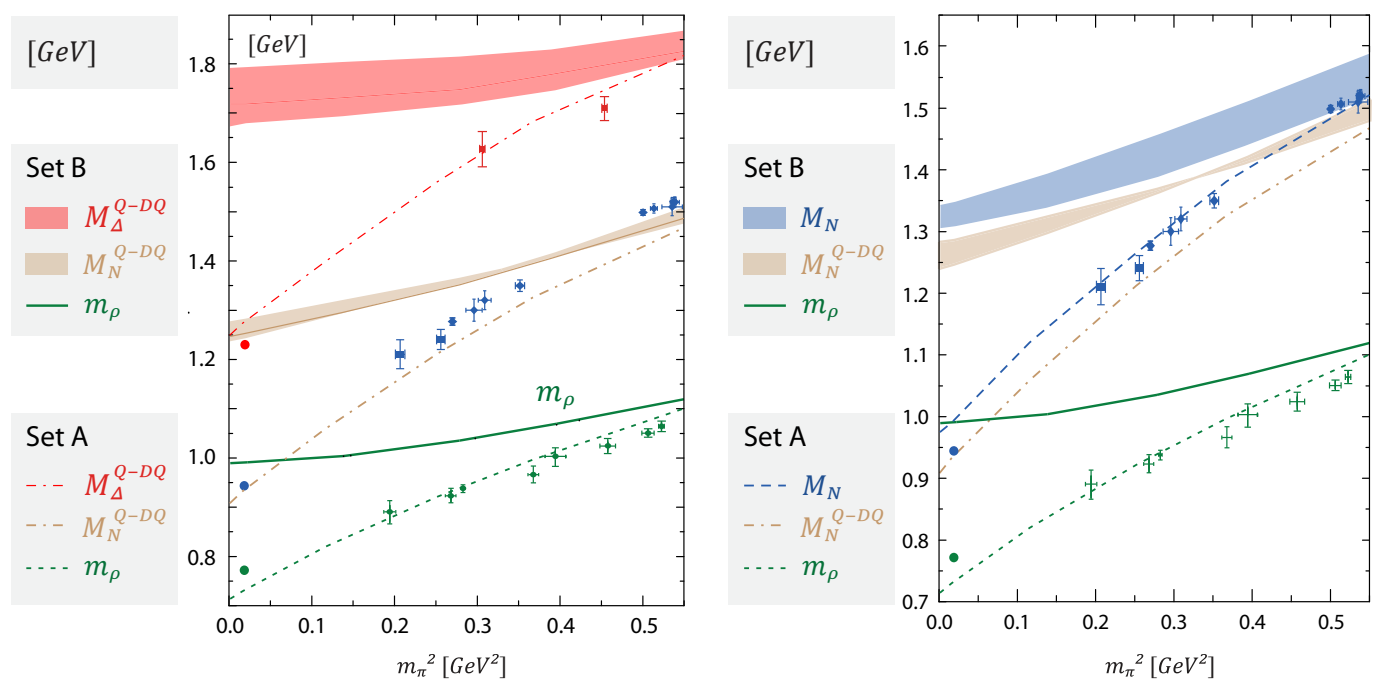

Fig. 3. (right panel adapted from Ref. ${ }^{[13]}$ ) Evolution with $m_{\pi}^{2}$ of $m_{\rho}, M_{N}$ and $M_{\Delta}$ compared to lattice data The left panel shows the quark-diquark model results for nucleon and $\Delta$ masses ${ }^{[8,11]}$; the right panel compares the quark-diquark and Faddeev results for the nucleon mass. Dashed and dashed-dotted lines correspond to setup A (i.e. $f_{\pi}$ is fixed as input); the solid line for $m_{\rho}$ and the bands for $M_{N}$ and $M_{\Delta}$ are the results of setup B (advertent inflation of hadronic observables to leave room for pionic corrections), where the variation w.r.t. to the interaction width parameter is explicitly taken into account to provide error estimates. Dots denote the experimental values.

and axial-vector diquark correlations that interact with the remaining quark provides a close approximation to the consistent three-quark nucleon amplitude.

By construction, the rainbow-ladder truncation omits all mesonic dressings of baryons. As pionic effects are certainly important for all hadrons this leads to the idea of changing the effective interaction such to advertently inflate the $\rho$ mass (Set B in Fig. 3) and thus to leave room for pionic corrections at small pion masses. It can be seen that such a treatment effects also the baryon masses in a way which provides room for further attraction by pions.

\section{$5 \quad$ Nucleon Electromagnetic Form Factors}

In order to calculate the nucleon's electromagnetic form factors in the given approach one has to relate the nucleon's electromagnetic current to the underlying description of the nucleon as a composite object. To this end the baryon must be resolved into its constituents to each of which the current can couple. A systematic procedure for the construction of a hadron-photon vertex based on electromagnetic gauge invariance $[\underline{\underline{41}}, \underline{\underline{42}}, \underline{43}]$ yields for the current operator

$$
J^{\mu}=-\bar{\Psi}_{f}\left(G_{0}^{\mu}+G_{0} K^{\mu} G_{0}\right) \Psi_{i},
$$

where $G_{0}$ denotes the product of dressed propagators and $K$ the kernel which appears in the respective bound-state equation. In the quark-diquark model, the incoming and outgoing baryon states are described by quark-diquark amplitudes $\Phi_{i}, \Phi_{f}$. Upon interaction with the external current, the baryon is resolved into its constituents: quark and diquark and the interaction between them. To each of these elements the current couples $[\underline{34}, \underline{44}$.

Previous nucleon form factor studies performed within the quark-diquark model $[\underline{30}, \underline{\underline{31}}, \underline{\underline{44}}, \underline{45}, \underline{\underline{46}}, \underline{\underline{47}}]$ share some common caveats. First, as already indicated above pionic contributions play an important role in the low-energy and small-quark mass behavior of the nucleon's electromagnetic structure. Such effects are not included in a quark-diquark 'core' and must be added on top of it $[32, \underline{48}, \underline{49}, \underline{50]}$. Second, access to the large- $Q^{2}$ region and thereby to the truly perturbative domain is so far only feasible upon implementing pole-free model propagators which, in turn, exhibit essential singularities at timelike infinity, cf. ref. ${ }^{51]}$. The problem of taking into account the realistic analytic structure of the quark propagator is not of fundamental concern; it merely awaits a thorough numerical treatment. Third, the quark-mass dependence of magnetic moments and charge radii, while emerging naturally in lattice calculations, is practically inaccessible in a quark-diquark model due to 

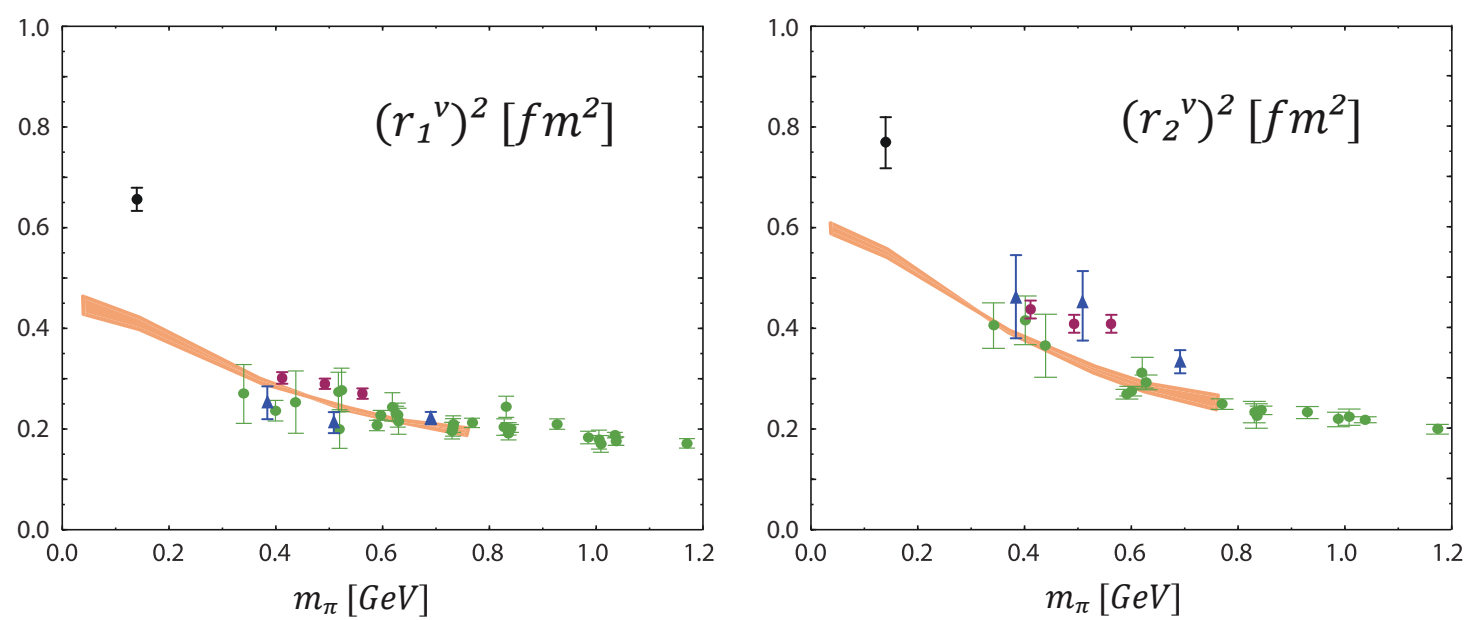

Fig. 4. (adapted from Ref. ${ }^{[9]}$ ) Quark-diquark model results for the squared isovector radii (corresponding to the Dirac and Pauli form factors $\left.F_{1,2}^{v}=F_{1,2}^{p}-F_{1,2}^{n}\right)$ in setup B, compared to lattice results ${ }^{[39,40]}$.

the unknown mass dependence of the modeled ingredients.

The rainbow-ladder based quark-diquark approach described in Section 3 removes the latter obstacle. Upon resolving the diquarks' substructure, the form factors are immediately related to the parameters in the effective quark-gluon coupling $\alpha\left(k^{2}\right)$, in particular: its quark-mass dependent coupling strength. Here we restrict ourselves to the quark 'core model' which represents a quark-diquark core that needs to be dressed by meson-cloud effects. A comparison of the core's static properties with lattice results is appropriate at larger quark masses; form factors depending on the photon momentum may be compared to experiment at $Q^{2} \gtrsim 2 \mathrm{GeV}^{2}$ where pioncloud effects are diminished.

The nucleon's Dirac and Pauli radii $r_{1}$ and $r_{2}$ are shown in Fig. 4 and follow a similar pattern as the pion charge radius ${ }^{[18]}$ : they are weakly dependent on the width parameter of the interaction and agree with lattice data at larger quark masses where the 'quark core' becomes the baryon. A natural feature of a quark-diquark model is the negativity of $F_{1}^{n}\left(Q^{2}\right)$. The presence of an axial-vector $d d$ diquark correlation centers the $d$-quark in the neutron and induces $r_{1}^{u}>r_{1}^{d}[11]$.

Only the components transverse to the photon momentum, i.e. those not constrained by current conservation, determine the physical form factor content. The respective contribution to the quark-photon vertex is known from its inhomogeneous Bethe-Salpeter solution ${ }^{[52]}$ and includes a $\rho$-meson pole which amounts to $\sim 50 \%$ of both pion and nucleon squared charge radii. The available information on those parts at larger $Q^{2}$, especially for the diquark-photon ingredients, is limited within the scope of the current approach; however they are mandatory to enable a realistic $Q^{2}$-evolution of the form factors and the proton's form factor ratio $\mu_{p} G_{E}^{p} / G_{M}^{p}{ }^{[11]}$. It is noteworthy that such uncertainties will be removed upon extending the the quark-diquark approach to a threebody framework: in a rainbow-ladder truncation, the analogue of Eq. (3) only involves the dressed-quark photon vertex and a dressed gluon propagator, i.e., quantities which are known and need not be modeled.

\section{Summary and Outlook}

In Landau gauge QCD, the Green functions of quarks and gluons are sufficiently well determined such that they can be used as a reliable input in relativistic bound state equations. These provide then a unified approach to mesons and baryons within a quantum-field-theoretical framework. Within the rainbow-ladder truncation meson observables and the nucleon mass have been calculated. These recent results mark a milestone in the effort to determine nucleon observables in a 'first-principle' functional approach to continuum QCD.

Electromagnetic properties of the nucleon and other baryons are currently investigated within this truncation scheme. The extensions of recent studies of meson properties beyond rainbow-ladder ${ }^{[12]}$ to the covariant Faddeev equations will improve on the now existing unified approach to meson and baryon properties. 
$R A$ thanks the organizers of the 5 -th International Conference on Quarks and Nuclear Physics for the invitation and for their support. He is thankful to Yuxin Liu and Huan Chen for their hospitality.

We are grateful to C.S. Fischer, M. Schwinzerl, and $R$. Williams for useful discussions.

This work was supported by the Austrian Science Fund FWF under Projects No. P20592-N16, P20496N16, and Doctoral Program No. W1203, and in part by the European Union (HadronPhysics2 project "Study of strongly interacting matter").

\section{References}

1 Fischer C S and Pawlowski J M 2009 Phys. Rev. D 80 025023 (Preprint 0903.2193)

2 Fischer C S, Maas A and Pawlowski J M 2008 Annals Phys. 324 2408-2437 (Preprint 0810.1987)

3 Alkofer R, Fischer C S, Llanes-Estrada F J and Schwenzer K 2009 Annals Phys. 324106 (Preprint 0804.3042)

4 Alkofer R, Fischer C S and Llanes-Estrada F J 2005 Phys. Lett. B 611 279-288 (Preprint hep-th/0412330)

5 Alkofer R and Greensite J 2007 J. Phys. G 34 S3 (Preprint hep-ph/0610365)

6 Alkofer R, Fischer C S and Llanes-Estrada F J 2008 Mod. Phys. Lett. A 231105 (Preprint hep-ph/0607293)

7 Eichmann G 2009 Hadron properties from QCD boundstate equations $\mathrm{Ph} . \mathrm{D}$. thesis University of Graz (Preprint 0909.0703)

8 Nicmorus D, Eichmann G, Krassnigg A and Alkofer R 2009 Phys. Rev. D 80054028 (Preprint 0812.1665)

9 Eichmann G, Alkofer R, Krassnigg A and Nicmorus D 2008 PoS Confinement8 077 (Preprint 0812.3183)

10 Nicmorus D, Eichmann G, Krassnigg A and Alkofer R 2008 PoS Confinement8 052 (Preprint 0812.2966)

11 Eichmann G, Cloet I C, Alkofer R, Krassnigg A and Roberts C D 2009 Phys. Rev. C 79 012202(R) (Preprint 0810.1222)

12 Williams R, these proceedings; Fischer C S and Williams R 2009 Phys. Rev. Lett. 103122001 (Preprint 0905.2291)

13 Eichmann G, Alkofer R, Krassnigg A and Nicmorus D 2009 (Preprint 0912.2246)

14 Krassnigg A 2009 Phys. Rev. D 80114010 (Preprint 0909.4016)

15 Maris P, Roberts C D and Tandy P C 1998 Phys. Lett. B 420 267-273 (Preprint nucl-th/9707003)

16 Holl A, Krassnigg A and Roberts C D 2004 Phys. Rev. C 70 042203(R) (Preprint nucl-th/0406030)

17 Maris P and Tandy P C 1999 Phys. Rev. C 60055214 (Preprint nucl-th/9905056)

18 Eichmann G, Alkofer R, Cloet I C, Krassnigg A and Roberts C D 2008 Phys. Rev. C $\mathbf{7 7}$ 042202(R) (Preprint 0802.1948)

19 Hess M, Karsch F, Laermann E and Wetzorke I 1998 Phys. Rev. D 58111502 (Preprint hep-lat/9804023)

20 Wetzorke I and Karsch F 2000 (Preprint hep-lat/0008008)

21 Orginos K $2006 \quad P o S \quad$ LAT2005 054 (Preprint hep-lat/0510082)

22 Liu Z and DeGrand T 2006 PoS LAT2006 116 (Preprint hep-lat/0609038)
23 Alexandrou C, de Forcrand P and Lucini B 2006 Phys. Rev. Lett. 97222002 (Preprint hep-lat/0609004)

24 Babich R et al. 2007 Phys. Rev. D 76074021 (Preprint hep-lat/0701023)

25 Maris P 2002 Few-Body Syst. 32 41-52 (Preprint nucl-th/0204020)

26 Maris P 2004 Few-Body Syst. 35 117-127 (Preprint nucl-th/0409008)

27 Anselmino M, Predazzi E, Ekelin S, Fredriksson S and Lichtenberg D B 1993 Rev. Mod. Phys. 65 1199-1234

28 Jaffe R L 2005 Phys. Rept. 409 1-45 (Preprint hep-ph/0409065)

29 Alkofer R, Kloker M, Krassnigg A and Wagenbrunn R F 2006 Phys. Rev. Lett. 96022001 (Preprint hep-ph/0510028)

30 Hellstern G, Alkofer R, Oettel M and Reinhardt H 1997 Nucl. Phys. A 627 679-709 (Preprint hep-ph/9705267)

31 Oettel M, Alkofer R and von Smekal L 2000 Eur. Phys. J. A 8 553-566 (Preprint nucl-th/0006082)

32 Alkofer R, Holl A, Kloker M, Krassnigg A and Roberts C D 2005 Few-Body Syst. 37 1-31 (Preprint nucl-th/0412046)

33 Höll A, Alkofer R, Kloker M, Krassnigg A, Roberts C D and Wright S V 2005 Nucl. Phys. A 755 298-302 (Preprint nucl-th/0501033)

34 Eichmann G, Krassnigg A, Schwinzerl M and Alkofer R 2008 Annals Phys. 323 2505-2553 (Preprint 0712.2666)

35 Taylor J G 1966 Phys. Rev. 150 1321-1330

36 Boehm M and Meyer R F 1979 Annals Phys. 120 360-384

37 Loering U, Kretzschmar K, Metsch B C and Petry H R 2001 Eur. Phys. J. A 10 309-346 (Preprint hep-ph/0103287)

38 Eichmann G, Alkofer R, Krassnigg A and Nicmorus D 2009 (Preprint 0912.2876)

39 Alexandrou C, Koutsou G, Negele J W and Tsapalis A 2006 Phys. Rev. D 74034508 (Preprint hep-lat/0605017)

40 Gockeler M et al. 2007 (Preprint arXiv:0709.3370 [hep-lat])

41 Haberzettl H 1997 Phys. Rev. C 56 2041-2058 (Preprint nucl-th/9704057)

42 Kvinikhidze A N and Blankleider B 1999 Phys. Rev. C 60 044003 (Preprint nucl-th/9901001)

43 Kvinikhidze A N and Blankleider B 1999 Phys. Rev. C 60 044004 (Preprint nucl-th/9901002)

44 Oettel M, Pichowsky M and von Smekal L 2000 Eur. Phys. J. A 8 251-281 (Preprint nucl-th/9909082)

45 Bloch J C R, Roberts C D, Schmidt S M, Bender A and Frank M R 1999 Phys. Rev. C 60062201 (Preprint nucl-th/9907120)

46 Oettel M and Alkofer R 2003 Eur. Phys. J. A 16 95-109 (Preprint hep-ph/0204178)

47 Bloch J C R, Krassnigg A and Roberts C D 2003 Few-Body Syst. 33 219-232 (Preprint nucl-th/0306059)

48 Hecht M B et al. 2002 Phys. Rev. C 65055204 (Preprint nucl-th/0201084)

49 Oettel M and Thomas A W 2002 Phys. Rev. C 66065207 (Preprint nucl-th/0203073)

50 Cloet I C, Eichmann G, El-Bennich B, Klahn T and Roberts C D 2009 Few Body Syst. 46 1-36 (Preprint 0812.0416)

51 Alkofer R, Ahlig S, Fischer C S, Oettel M and Reinhardt H 2000 Nucl. Phys. A 663 683-686 (Preprint hep-ph/9907563)

52 Maris P and Tandy P C 2000 Phys. Rev. C 61045202 (Preprint nucl-th/9910033) 